\title{
Le rôle du chœur dans les sept devant Thèbes
}

Marie Delcourt

Citer ce document / Cite this document :

Delcourt Marie. Le rôle du chœur dans les sept devant Thèbes. In: L'antiquité classique, Tome 1, fasc. 1-2, 1932. pp. 25-33; doi : 10.3406/antiq.1932.2936

http://www.persee.fr/doc/antiq_0770-2817_1932_num_1_1_2936

Document généré le 24/01/2017 


\title{
Le rôle du chour \\ dans “ Les Sept devant Thèbes ”.
}

\author{
par Marie Delcourt.
}

Les Sept devant Thèbes sont une pièce difficile. Wilamowitz, dans ses Aischylos Interpretationen, a bien marqué le nœud de la difficulté : la première partie de la pièce a pour sujet la défense de Thèbes par Etéocle, "celui dont la gloire est bien méritée "; à la fin, tout l'intérêt s'attache à la malédiction qui frappe la race de Laïos. Le drame doit son unité au personnage d'Etéocle, à la fois rempart et fléau de Thèbes. M. Mazon, dans sa préface aux Sept, insistant sur la beauté du caractère d'Etéocle, " héros qui est aussi un maudit ", voit une richesse psychologique où Wilamowitz voit une succession. Entre le début et la fin de l'œuvre, il y a certes plus d'unité que Wilamowitz ne consent à le reconnaître. Mais pour pouvoir dire, comme le fait M. Mazon, que "la figure d'Etéocle est la plus belle à coup sûr de tout le théâtre grec ", il faut laisser dans l'ombre toute l'explosion de haine du frère contre le frère et $s$ 'en tenir à l'admirable invocation du début, où le roi de Thèbes prie la Malédiction paternelle de le frapper seul et d'épargner la ville (69 sqq.). Cela revient à dire que la complexité du caractère se développe dans le temps et non dans la simultanéité, ce qui nous ramène, à peu de chose près, à la remarque de Wilamowitz.

Peut-être la pièce et son personnage central seraient-ils plus intelligibles si l'on précisait mieux quel y est le rôle du chœur. Tous les critiques marquent l'intérêt de la scène où les Thébaines apparaissent épouvantées, tandis qu'Etéocle couvre d'injures ces semeuses de panique. Mais on ne s'est guère demandé ce que fait le chœur dans le reste de la tragédie. 
De qui est-il composé? De jeunes filles, dit-on dans la plupart des éditions, en s'appuyant sur les deux passages où il prie les dieux d'écouter les prières des vierges. Il est bien certain que les femmes semblent parler d'elles-mêmes lorsqu'elles disent: "I $\delta \varepsilon \tau \varepsilon \pi \alpha \varrho \theta \varepsilon v^{\prime}(1) v$

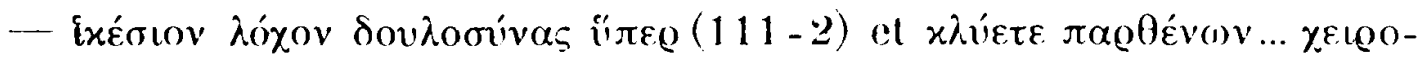
tóvous $\lambda$ itcis (171).

Plus loin, on a interprété de la même façon le passage suivant:

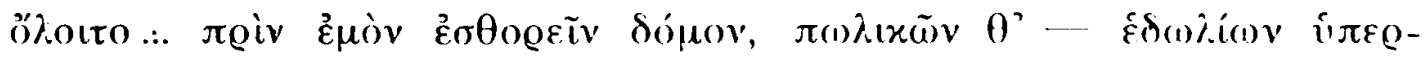

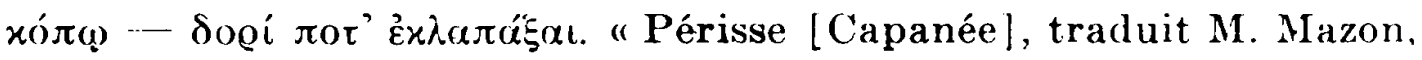
avant qu'il ait pu faire irruption dans ma demeure, et, de sa lance arrogante, me jeter hors de ma chambre virginale". Mais $\vec{\varepsilon} \gamma \hat{\lambda} \alpha \pi(i-$ $\zeta \varepsilon \iota v$, ne se trouve qu'ici et dans une construction sans analogue.

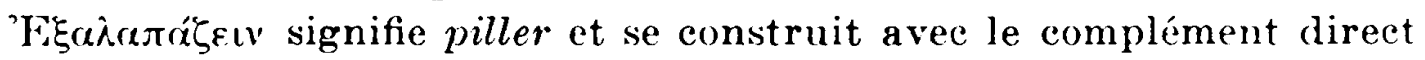
du contenant pillé. Hermann, qui comprenait comme M. Mazon. trouvait l'ellipse trop dure et insérait $\left\langle\mu^{\prime}\right\rangle$ après $\dot{\varepsilon} \delta(\omega) \lambda^{\prime}(\omega) v^{\prime}$. M. Mazon n'admet pas l'insertion et tire $\mu \varepsilon$ de $\varepsilon j \mu o ́ v$ qui précède. On se demande si une jeune fille grecque dirait " $m:$ maison " en parlant de celle de ses parents. Ne vaudrait-il pas mieux comprendre: "Avant qu'il ait pu faire irruption dans ma maison et, de sa lance arrogante, mettre à sac les chambres des jeunes filles" ?

Il reste done deux passages indiscutables où les paroles du chøur donnent à penser que l'on entend des jeunes filles. Mais, si l'on admet cela, on est aussitôt frappé par dos invraisemblances psychologiques qu'il faut relever.

Il est inadmissible que des jeunes filles appellent Etéocle "en-

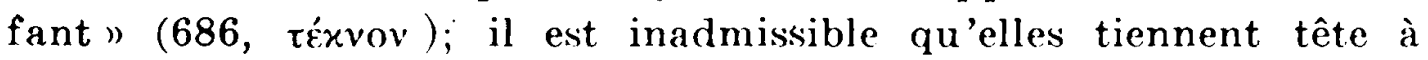
leur roi comme on le voit après la violente tirade d'Etéocle sur la lâcheté des femmes. Il est difficile d'admettre que des jeunes filles aient assez d'autorité pour protester, comme le fait le chœur, contre la résolution d'Etéocle d'aller combattre Polynice et pour discuter avec lui sur la nature même de la Malédiction. Cela est si évident que M. Mazon, rompant avec une tradition vénérable, dit que le chœur est composé de femmes, sans spécifier. Si l'on ne consultait que la psychologie du drame, on se les représenterait vieilles. $\mathrm{Ne}$ disent-elles pas (293) que leur angoisse est celle de colombes qui craignent pour leur couvée? L'hypothèse la plus satisfaisante, capable de concilier le sens profond du drame avec les deux passages où il est question des jeunes filles en prières serait celle-ci : le chour est composé de vieilles femmes accompagnées d'un groupe 
de très jeunes filles, qui tiennent des rameaux, mais ne chantent pas. Les vieilles les désignent aux dieux, parce que le viol des vierges est particulièrement capable de provoquer la colère divine. On se souvient de la légende de Cassandre et d'Ajax, pour lequel il y avait, il est vrai, des circonstances aggravantes. Le coryphée est une vieille femme. Cela n'aurait rien de contraire aux habitudes d'Eschyle: dans les Suppliantes, le chœur est composé de princesses et de suivantes; le coryphée parle au nom des premières seules. Quant à l'idée de prendre la captivité des jeunes filles et des vieilles femmes comme symbole de ce que la guerre a de plus affreux, elle est naturelle: on se souvient du parti qu'Euripide en tire dans les Troyennes, où la vieille Hécube et un groupe de jeunes filles l'esclavage sous sa forme la plus pitoyable - restent en scène pendant toute la pièce, pour encadrer Cassandre, Andromaque, Hélène, personnes agissantes et différenciées.

Revenons au texte. Wilamowitz a bien marqué que la parodos est une sorte d'anticipation: l'armée argienne n'est pas encore aux portes et les Thébaines ne peuvent entendre que de loin le grincement des chars et le bruit des sabots. Leur exagération explique la colère d'Etéocle et sa violente sortie contre les femmes. Les commentateurs, frappés du contraste entre tant d'épouvante et une si ferme résolution, n'ont peut-être pas assez remarqué.ceci : le chœur ne paraît nullement humilié par les injures d'Etéocle. Il se justifie : un danger exceptionnel provoque une terreur exceptionnelle et impose un recours exceptionnellement pressant à la divinité. Ici se marque, entre les deux adversaires, une première différence dans leur façon de prendre la situation présente; "Prie les dieux, dit Etéocle, mais sans te laisser lâchement aller. La discipline, femme, engendre le succès sauveur. Voilà le mot d'ordre. - Oui, mais Dieu peut davantage encore. Souvent, il redresse en plein malheur celui qui, sans recours, sombrant dans l'inextricable détresse, ne voyait plus que brouillard devant ses yeux. " Sous cette forme, l'antithèse paraît simplement une banale affirmation de piété religieuse corrigeant une affirmation trop hardie de l'homme qui met sa confiance en soi. Plus tard, elle se précisera.

Cependant, l'effroi reprend les femmes et la colère Etéocle, qui dit :

"Ah, Zeus, qu'as-tu créé en nous donnant la race des femmes? Et le coryphée répond vertement: 
"Une race pitoyable, et celle des mâles tout autant, une fois la ville prise."

Réplique impossible dans une bouche de jeune fille, réponse empreinte d'une expérience qui fait penser (encore une fois) à la philosophie dóșabusée de la vieille Hécube dans les Troyennes.

"Aie donc plus de confiance dans les dieux que tu pries, dit le roi, et cesse d'effrayer ceux qui t'entourent ", à quoi le coryphée répond par ce vers :

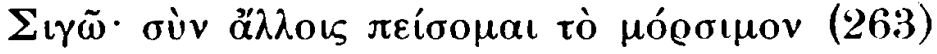

"Je subirai avec d'autres ce que le sort voudra ". Résignation qui

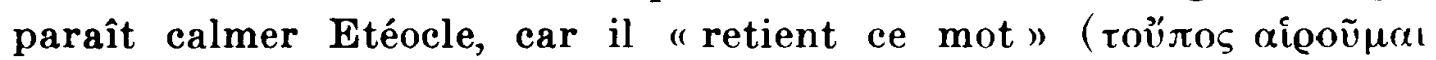

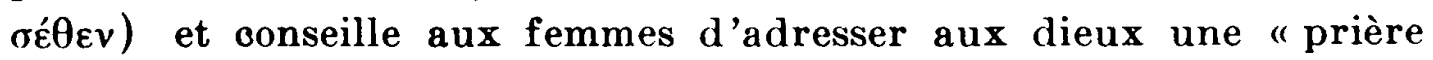
meilleure " que leurs supplications affolées, à savoir qu'ils veuillent combattre avec lui ( l'épisode donne l'impression suivante: les femmes craignent autre chose que ce que redoute le roi. Elles essaient d'apaiser les dieux qu'Etéocle cherche simplement à mettre du côté des combattants cadméens. Au surplus, la colère et les exhortations du roi n'obtiennent rien d'elles. Dans le premier stasimon, elles reprennent exactement les mêmes thèmes qu'elles ont traités dans la parodos, long

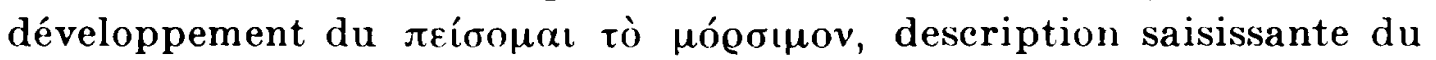
sort lamentable de la ville prise. De telles paroles conviennent certes mieux à des femmes d'âge qu'à des jeunes filles. Mais il arrive souvent que des chœurs de tragédie expriment une sagesse, une expérience purement humaines et il ne faut pas vouloir trop tirer de ce genre d'indications.

La scène des deux fois sept chefs est construite symétriquement: un exposé du messager, une réplique d'Etéocle, un commentaire chanté du chœur qui prie les dieux de rejeter la punition sur les Argiens coupables de démesure. Mais la banalité cesse brusquement lorsqu'Etéocle annonce qu'il va se porter à la rencontre de son frère.

C'est ici que commence la seconde partie de la tragédie, celle qui a pour enjeu moins le sort de Thèbes que la malédiction des Labdacides ( ${ }^{1}$. Ici commence aussi le véritable conflit entre Etéocle et

(') Etéocle porte à la fois la malédiction d'Apollon, lancée sur toute la race de Laïos et l'imprécation d'Oedipe contre ses fils. Dans ce qui suit, il est inutile de faire la distinction entre les deux. On nous permettra de les désigner par l'un ou l'autre nom. 
le chœur. Il tient en quelques répliques (vers 686-719), mais si char gées de sens qu'il est impossible de ne pas voir ici le centre de ic pièce.

Etéocle tient pour fatale la malédiction qui pèst sur lui :

“Puisque c'est Dieu qui précipite les choses, qu'elle aille, chassée par la tempête, vers son lot, la vague infernale, cette race de Laïos häe d'Apollon ).

Ise chœur n'accepte pas cette explication. Etéocie peut, s'il le veut, refuser à l'égarement l'entrée de son âme. S'il cède, c'est folie pure:

"Quel est ce délire, enfant? Ne laisse pas l'égarement d'une folie meurtrière emplir ton cour et t'emporter. Rejette déjà en son principe cette convoitise mauvaise" (trad. Mazon, 686-8).

Et quand Etéocle allègue l'Imprécation qui lui conseille de mourir le plus tôt possible, puisqu'en tout cas il faut mourir, le chœur proteste: "Mais c'est à toi de ne pas te laisser entraîner! Est-ce que l'Erinys ne quitte pas une maison quand les mains des hommes tendent aux dieux des offrandes agréées?"

M. Croiset, dans son livre récent sur Eschyle, se demande si la question du libre arbitre s'est posée à l'esprit du poète et il estime téméraire d'y répondre affirmativement. Les quarante vers des Sept qui résument toute la substance psychologique de la tragédie sont d'une telle précision que toute hésitation paraît cependant impossible. Certainement, Eschyle s'est interrogé sur l'efficacité du vouloir humain; il pense, comme le chœur, que la volonté peut prévaloir sur une Imprécation, que celui qui, sans résister, s'abandonne à tine Imprécation est un fou. De plus, il a réfléchi sur ce problème d'une façon pénétrante et l'analyse qu'il en donne est d'une finesse qui ne laisse rien à souhaiter.

Il sait que le moment pendant lequel nous pouvons résister à l'Até est extrêmement court. C'est pourquoi les femmes adjurent Etéocle de ne pas se laisser emporter par cette folie qui, dès qu'elle envahit le cœur, le remplit $(\theta v \mu o \pi \lambda \eta \theta \hat{\eta} \varsigma)$. Nous devons rejeter le

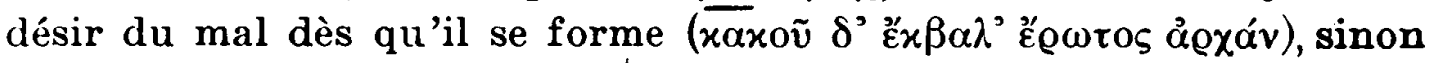
il risque d'être plus fort que la bonne volonté. L'acte mauvais est donc le résultat d'une collaboration entre le dieu et l'homme. C'est le dieu irrité qui, soit caprice, soit châtiment - sur ce point, il nous est impossible de savoir quelle est, dans les Sept, la version suivie par Eschyle - inspire à l'homme le désir du mal. Ici se place 
la tragique minute de l'option. L'homme peut vaincre, offrir aux dieux des offrandes qui soient acceptées et attendre un revirement du Daimon (705). Mais, s'il cède à l'Até, alors les dieux accéléreront le mouvement et précipiteront sa chute en achevant de l'égarer. Eschyle, qui s'est beaucoup interrogé sur l'efficacité du vouloir humain, présente ici les deux thèses. Etéocle se retranche derrière un fatalisme absolu:

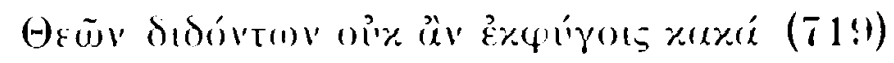

qui masque sa haine et lui permet le fratrieide. Le chour, évidemment interprète du poète, tient pour la liberté de l'homme et le dit le plus clairement du monde.

Du reste, le moment n'est pas encore venu où un dramaturge construit toute une tragédie sur le choix d'un homme qui hésite entre deux partis. Etécle n'a pas hésité et, dés qu'il s'en va vers la septième porte, le chour sait que la malédiction est accomplie; le roi a laissé passer la minute de l'option : l'Erinys qu'il aurait pu retenir est désormais déchainée, il n'y a plus qu'à attendre le coup. Tout le conflit tragique tient en quarante vers. Plus tard, les poètes sauront mieux ralentir un débat, suspendre une décision pour les rendre plus pathétiques. Encore n'est-ce pas un art qu'ils apprencloont facilement. Entre l'hésitation oratoire de Médée (") mess enfants, mes enfants...") et l'hésitation vraie d'Agamemnon et d'Iphigénie qui fait le tissu même de la clernière pièce d'Euripide, il y a vingtcinq ans de floraison tragique. Eschyle, qui sait si bien opposer deux thèses, ne se soucie pas encore beaucoup de montrer comment l'une d'elles peut conquérir ou même entamer l'adversaire. Indifférence poussée à l'extrême dans les Sept, car, dans les Suppliantes, Pélasgos discute avec les princesses et estime finalement que son devoir est de les secourir. lci, le roi et le chœur restent rigoureusement étrangers l'un ì l'autre. Pas plus qu'Etéocle n'a pu rassurer les Thébaines effrayées, pas plus elles ne peuvent le faire douter de son droit à tuer son frère. Aucun échange entre les deux adversaires.

Le second stasimon est écrit de façoņ à donner raison à ceux qui voient dans la tragédie deux actions soudées par la présence d'un héros unique. Ises femmes ne parlent plus que de la Malédiction. Elles semblent avoir oublié leurs terreurs et les menaces de la défaite pour ne s'intéresser qu'au problème moral de la colère divine. Elles n'ont plus d'espoir pour les frères emnemis: "Le fer a décidé 
qu'ils oceuperaient de leurs terres juste ce qu'en tiennent deux morts $\left({ }^{2}\right)$ ", mais cela n'a rien à voir avec le sort de Thèbes qui paraît les laisser indifférentes. Ise messager leur annonce d'abord que Thèbes est sauvée, puis que les deux princes se sont entretués.

Ici vient une notation curieuse, qui n'a peut-être pas été assez mise

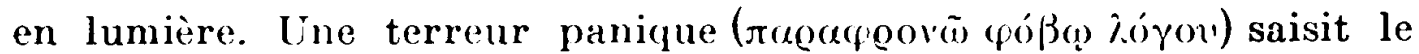
chour lorsqu'il apprend le double meurtre et, à part quelques mots pour se féliciter de la victoire $(822-826)$, le reste de la pièce n'est qu'une lamentation funéraire. Cela nous éclaire rétrospectivement sur la nature exacte de l'épouvante qui règne parmi les Thébaines au début de la pièce. Ce qu'elles redoutent, c'est à la fois la ville prise et la malédiction des Labdacides. Si elles craignaient uniquement la défaite et l'esclavage, la frayeur ne les reprendrait pas alors qu'elles savent ces deux maux conjurés. C'est parce qu'elles tremblent devant les suites de l'imprécation qu'Etéocle n'arrive pas à les calmer. Un chef qui est un maudit peut être brave, sa présence n'arrive pas à rassurer. C'est pour cela qu'elles lui rappellent les dieux et la nécessité de prendre leur conseil. C'est pour cela qu'il leur faut un grand effort pour consentir à se solidariser avec lui. I'attitude du chœur dans la première partie est incompréhensible si l'on n'admet qu'il est dominé par la pensée de la malédiction et si l'on ne sent latent le conflit entre des femmes pieuses et optimistes d'une part, et, d'autre part, un roi qui allègue la fatalité pour pouvoir s'abandonner à sa haine.

On objectera que cela n'est pas dit, puisqu'avant le second épisode il est à peine question de la Malédiction et que le chœur, en tous cas, n'en parle pas. Assurément, le raccord entre les deux thèmes est gauchement fait, et cependant Eschyle a essayé de le marquer. Quand le messager arrive après la victoire, il rassure ironiquement les choreutes, "filles élevées par vos mères". Ce vers ne s'explique que comme rappel des railleries d'Etéocle au premier épisode, car lor'squ'entre le messager, le chceur vient d'exprimer sa terreur, non pour son propre sort (dont il ne paraît plus avoir grand souci à partir du second épisode), mais pour le sort des deux princes, erainte si peu chimérique qu'elle est déjà réalisée. Nul doute qu'ici Eschyle n'ait voulu renoner avec le début de la tracédie,

(2) Cette image (731) reprise un peu loin (817) fait penser à l'admirable conte de Tolstoï, Ce qu'il faut de terre à un homme. 
tout rempli par l'affolement des femmes. En revanche, il ne s'est nullement soucié, dans la première partie, de préparer ses auditeurs au rôle qu'allait jouer le chœur. C'est que, pour un poète qui apprend son art, et, à plus forte raison, pour un poète qui crée son art, il est plus facile de rappeler que de préparer.

En face d'Etéocle heureux de réaliser la malédiction familiale parce qu'elle lui permet de tuer son frère, les 'Thébaines représentent done la croyance eschyléenne en la bonne volonté humaine capable de vaincre L'Egarement. Le conflit entre lui et elles est certainement la partie la plus tragique du drame; il tient en quelques vers d'une exceptionnelle plénitude, d'une exceptionnelle riehesse de sens.

Si la parodos et le premier stasimon peuvent être mis dans la bouche de jeunes filles, toutes les parties dialoguées - c'est-à-dire tout ce qui traduit le duel entre le roi et ses sujettes - ne sont concevables que si elles sont dites par des femmes mûries par l'expérience et qui parlent avec autorité. Encore une fois, tout s'explique si l'on imagine quelques jeunes filles parmi les choreutes, encadrées par des vieilles femmes au nom de qui parle le coryphée.

Qu'il y ait du flottement dans la psychologie du chœur, cela est bien certain: sinon, devrait-on s'interroger si longuement sur sa véritable personnalité? Tout serait plus clair pour nous s'il nous disait explicitement, au début, que la malédiction des Labdacides le fait trembler autant et plus que la menace d'esclavage, et que c'est à cause de l'imprécation d'CEdipe qu'il lui est si difficile de se solidariser complètement avec Etéocle. Et la pièce aurait plus d'unité si, après des cris d'épouvante qui sont censés $n$ 'avoir d'autre rai. son que le danger qui plane sur Thèbes, on sentait mieux la détente de la délivrance.

Le chœur semble donc tenir deux rôles successifs, parce qu'il contrebalance les deux aspects d'Etéocle: les Thébaines sont d'abord opposées au roi comme des faibles à un chef courageux, plus tard comme des êtres raisonnables et pieux en face d'un homme abandonné à la haine.

Indépendamment de cette raison, il faut remarquer que, dans plusieurs pièces d'Eschyle, la personnalité du chœur échappe à une définition unique. Les Océanides parlent successivement comme des déesses marines bien au courant de ce qui se passe dans le monde élémentaire, puis en sages petites Athéniennes désireuses ne ne pas se narier au-dessus de leur rang, enfin comme des héroïnes qui bra- 
vent Zeus et se sacrifient pour mourir avec Prométhée. La scène du tombeau dans les Choéphores serait plus satisfaisante pour nous si c'était le Sénat d'Argos qui rappelait aux enfants d'Agamemnon leur devoir envers leur père: des esclaves, des étrangères qui ignorent tout des traditions de la maison ont peu qualité pour tenir un si grave langage. Dans la seconde partie des Sept, un chœur de vieillards aurait eu autorité pour rappeler Etéocle à la raison, mieux que des femmes qu'on vient de voir en plein affolement et violemment injuriées par leur roi.

En composant ses trilogies, Eschyle tenait certainement compte des voix et cherchait à varier les timbres: basses dans Agamemnon, soprani dans les Choéphores, alti dans les Euménides. Le peu que nous savons des ensembles trilogiques nous indique que les poètes ont gardé ce souci musical dans la composition des chœurs: voix de jeunes gens dans Alexandre, d'hommes faits dans Palamède, soprani dans les Troyennes. Dans la poésie tragique, la musique n'était pas une esclave et elle dictait des choix dont la raison échappe à nous qui, du poème chanté, ne connaissons plus que les paroles. C'est pourquoi il est toujours imprudent de faire des conjectures sur la composition d'un chour perdu en tenant compte uniquement des convenances du sujet. M. Croiset admet que, dans les deux pièces qui suivaient les Suppliantes, c'étaient encore les princesses égyptiennes qui formaient le chœur. Rien de moins vraisemblable qu'une telle supposition, qui donnerait à tout un ensemble trilogique un accompagnement musical d'une inévitable monotonie. 